\title{
IMPROVING PRICE/PERFORMANCE RATIO OF A LINEAR MOTOR BY MEANS OF LEARNING CONTROL
}

\author{
Bas J. de Kruif* Theo J.A. de Vries * \\ * University of Twente, Drebbel institute of Mechatronics \\ P.O. Box 217, 7500 AE, Enschede, The Netherlands
}

\begin{abstract}
A set of experiments is performed to experimentally validate that Learning Feed-Forward Control can compensate for reproducible errors that were introduced because of a low-cost electro-mechanical construction. A linear motor is used in this set of experiments in which the magnet plates and the driving coils could be exchanged. Several configurations are tested with different combinations of magnet plates and coils while Learning Feed-Forward Control is used to compensate for the disturbing effects. It is shown that the tracking error after learning is hardly influenced by the accuracy of the placement of the magnets and the tolerance on their strength. This allows to use low-cost magnets in a linear motor without degenerating the performance. The quality of the driving coils has a more significant influence on the tracking error after learning.
\end{abstract}

Keywords: Learning Feed-Forward Control, Non-Repetitive movements, Synchronous Linear Motor, Cogging compensation

\section{INTRODUCTION}

The mechatronic design approach develops a system. The mechanical construction and the control of this system are not separated but seen as one in the design phase. It is kept constant in mind that a broad set of solutions leads to a system with the given specifications. Due to this integrated approach control and construction are interchangeable and this freedom, acquired by the mechatronic approach, can be used to fulfill a second objective, e.g. low-cost construction. This approach can be applied for the design of a linear motor. Instead of expensive construction, we investigate the possibility to reduce the cost of the constructional part of the motor and to compensate for the resulting loss of performance by means of learning control. The motor we investigate has

1 This research is supported by the Euregio "GermanDutch Mechatronic Innovation Centre" permanent magnets fixed on the stationary part. A three phase current has to be applied with a certain phase depending on the position and velocity of the translator to generate a thrust force. To increase this force, the coils in the translator are mounted on an iron comb to bundle the flux. The permanent magnets in the stationary part attract these iron cores, which results in cogging forces. The motor has preference positions due to this attraction and during a movement a periodic force is exerted on the translator. By skewing the permanent magnets this effect can be minimised (Gieras and Piech, 2000). However, to obtain the reduction of the cogging force by skewing the magnets, the set of magnets should be tightly toleranced and placed with high accuracy.

Accurate placement is a constructional expensive solution and is therefore unwanted. A possible solution to avoid this accurate placement is to identify the attraction force between the iron and 
the permanent magnets and apply a feed-forward force by the control mechanism to counteract the attraction force. This allows larger tolerances on the placement and the magnet strength, because the effect it would have is compensated for before introducing an error. Another expensive constructional solution is used to minimise the magnetic field present outside of the moving part. Generally, the coils are placed on a comb of iron to bound the flux within the coils as stated before. The outer-teeth don't contain coils and are merely used to minimise the magnetic field present next to the translator. This part of the magnetic field would interact with the permanent magnets, which would also result in an unwanted force. These outer-teeth are less desirable because they limit the stroke. Again, it would be profitable if the related disturbance force would be compensated for by a feed-forward controller such that these outer-teeth could be omitted without introducing an increased tracking error.

Implementation of the above approach requires identification of the forces that are to be compensated. This identification is time-consuming and, if it has to be done accurately, difficult. Furthermore, due to differences from plant to plant, this identification has to be repeated for each plant. However, Learning Feed-Forward Control (LFFC) is a method that identifies and simultaneously compensates these effects while the system is controlled (Velthuis, 2000). In (Yao and Xu, 2002) a similar problem is addressed from the adaptive control point of view. This results in a more theoretical framework. These methods could make it possible to compensate for loss of performance due to low-cost construction by adding feed-forward control.

In this paper the results of a set of experiments are presented in which LFFC was applied on several configurations of a linear motor. The configurations existed of the combination of a good and a bad set of magnets in combination with a comb in which the outer-teeth were included or omitted.

This paper is organised as follows: In section 2 the principle of learning feed-forward control is treated. The next section describes the experiments that have been executed. In section 4 the results are given and in the last section the conclusions will be drawn.

\section{LEARNING FEED-FORWARD CONTROL}

In this section the learning feed-forward control scheme (figure 1) is treated. More information can be found in (Velthuis, 2000). P is the plant, $\mathrm{C}$ is the controller, LFF is the learning feed-forward controller and $\mathrm{R}$ is the reference generator.

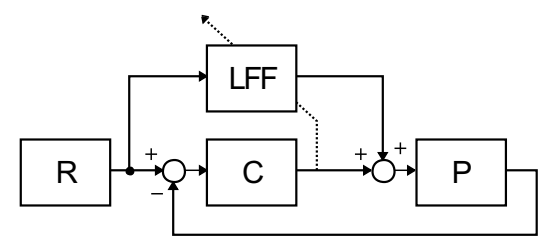

Fig. 1. The learning feed-forward control scheme

2.1 Working Principle

It can be seen from this figure that if the feedforward controller equals the inverse of the plant, the tracking error will be zero. This is the goal of LFFC: learn the (stable part of the) inverse of the plant. The inverse of the plant is approximated based on samples that are obtained during the control of the plant. A feedback controller is present to compensate for external disturbances like noise and mismatches between the feed-forward controller and the actual inverse of the plant.

A feed-forward controller acts on the reference signal and is not reacting on errors. This makes it possible to compensate for effects before they introduce an error. This is also the case in learning feed-forward control; the LFF controller generates a feed-forward signal based on the reference. The forces that are required to nullify (non-linear) state dependent effects like cogging and friction should be present in this feed-forward signal. For the LFFC to be able to compensate for these effects, it should have access to the reference states on which these effects depend. For instance, if the feed-forward controller should compensate for friction, it should have access to the velocity because friction depends on this variable. If the feed-forward controller should compensate for the influence of the (unknown) inertial load, it should have access to the acceleration.

If a feedback signal is still present after the feedforward signal is applied, an error between the desired and the actual LFF output is present due to an incorrect inverse. Hence, this feedback signal is a measure of how the feed-forward signal should be updated at that reference state (Otten et al., 1997). By adding a part of the filtered feedback signal to the already present feed-forward signal at the reference state, the error will decrease on the following occurrence of that state. The filtering is applied to omit effects above the bandwidth. If the error was due to noise with zero mean, the effect it has on the learnt inverse will disappear, because of averaging.

\subsection{B-spline network}

The actual function of the block LFF in figure 1 is function approximation; its goal is to determine 
a relation between several inputs and an output based on examples. The function approximator should approximate the required feed-forward signal for a given reference to compensate for the disturbing effects. In this paper a B-Spline Network (BSN) is used as a function approximator (Brown and Harris, 1994). This network is chosen for its simplicity. Other function approximators are also possible (de Kruif and de Vries, 2001).

A BSN is a neural network that uses B-spline basis functions to store an input-output mapping. A Bspline of order $n$ consists of piecewise polynomial functions of order $n-1$. In this research only $2^{\text {nd }}$ order B-splines will be considered. The function evaluation of a B-spline is generally called the membership and is denoted as $\mu$ (fig 2). That part of the input space for which $\mu$ is unequal to zero is called its support. To create an i/o mapping, B-splines are placed on the domain of the input of the BSN, in such a way that at each input value the sum of all memberships equals 1 . The output of the BSN is a weighted sum of the Bspline evaluations:

$$
u_{\mathrm{ff}}(r)=\sum_{i=1}^{N} \mu_{i}(r) w_{i}
$$

In which $w_{i}$ is the weight associated to the $i^{\text {th }}$ B-spline and $N$ is the number of B-splines.

The number of splines that is necessary to cover the input space grows exponentially with the dimension of the input space. If only $n$ splines were required for input dimension of one, $n^{2}$ splines are required for a dimension of two and $n^{3}$ splines for a dimension of three. The exponential growth of the number weights is called the curse of dimensionality (Brown and Harris, 1994; de Vries et al., 2001). The exponential growth of the number weights results in large memories, bad generalisation and difficult training.

\subsection{Parsimonious LFF}

One solution to overcome this curse of dimensionality is to use parsimonious LFF (de Vries et al., 2001). This solution uses several networks, each compensating for one effect and adding the output of these networks together. If these individual effects depend on less variables than the total input space, the individual networks have to learn a relation with fewer inputs reducing the total number of weights. Take for example the compensation of friction and cogging. The friction is velocity-dependent and can be approximated by $n$ splines while the cogging is position-dependent and can be approximated by $m$ splines. If these effects would be learnt in one two-dimensionally network, the number of weights would be equal to

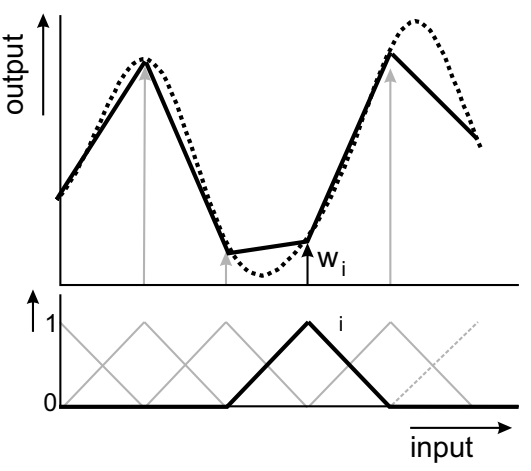

Fig. 2. Approximation of a function by a B-spline network. At the bottom of the figure the triangular functions denote the member ship functions of the splines. The dashed line is the actual function and the solid line in the top figure is its approximation

$n \times m$. However if both effect would be learnt in a separate network, the number of required weights would become $n+m$ instead of $n \times m$.

The training of a parsimonious network is rather difficult because there are several networks that have to be learnt but only one learning signal is present. I.e., there is a difference between the total sum of the networks and the inverse, but it is unknown which network introduces this difference. If a motion profile is used in which one effect is made dominant, the error can be contributed to this effect (de Vries et al., 2001). The creation of such motion profiles require a-priori knowledge. Generally the dominant effects are known in motion systems, but the magnitudes of these effects are unknown. The construction of dedicated motion profiles is done for the learning of the networks in this paper.

\section{EXPERIMENTS}

In the experiments we evaluate the error reduction achieved by LFFC for several configurations of a linear motor. The goal is to investigate if the tracking error enlargement due to low-cost construction can be overcome by LFF. The plates of magnets and the comb with coils could be exchanged in the motor we investigated. For the experiments two different plates of magnets were used. One plate had a tightly toleranced set of magnets that were placed accurately, and the other plate had magnets with large differences between the strengths and were badly placed. These plates will be referred to as the good and the bad magnet plates. Next to the different plates, two different combs with coils were used. One of these combs had its outer-teeth still present, while in the other comb these teeth were removed. The used feedback controller was a PD-controller, with fixed settings over all experiments. 
Several state-depending effects will have an influence on the tracking error. The dominant effects are cogging, friction, unknown mass, and an effect due to the removal of the outer-teeth.

Cogging: This is an position dependent effect. To compensate for this effect by feed-forward the controller needs to have access to the position.

Friction: The friction that is present is assumed to be only velocity-dependent. Hysteresis around $v=0[\mathrm{~m} / \mathrm{s}]$ is not compensated for, nor is position-dependent friction. The influence of the position dependent effects is expected to be small relative to the velocity-dependent influence.

Unknown mass: The mass of the moving part is unknown. The network that is responsible for the feed-forward signal due to the mass has to learn one parameter, the mass, and the input of this network is the acceleration.

Absence of outer-teeth: The magnetic field present next to the translator will interact with the permanent magnets resulting in a positiondependent force. Next to this, the generated force will be different if these outer-teeth are missing due to incorrect commutation. The commutation calculated by the controller is designed for a comb including the outer-teeth. The commutation of the motor currents is a function of the velocity and the position.

The above mentioned effects should be compensated for by the LFFC. To avoid the curse of dimensionality we only want to use one-dimensional B-spline networks. If a two-dimensional network would be used, proper training of the network is nearly impossible if at the same time a good accuracy is required. Only for the compensation of the removed outer-teeth a network is required that depends on position and velocity. For the compensation of the other effects one-dimensional networks are needed. Therefore, three one-dimensional networks are used and it is assumed that the velocityposition dependent part is small.

The training motions are chosen such that one effect is dominant during the motion. The network that should compensate for the dominant effect is the only network that will be adapted. The networks are adapted successively for several iterations until no error reduction takes place anymore. The first network to be trained is the acceleration net. This network should compensate for the unknown mass. To get an estimate of the mass, a motion is performed that will accelerate at several positions and several velocities. This is done to minimise the influence of the other effects. The acceleration as function of the time is given in figure 3 . The next network that is trained is the network that should compensate for the position-dependent effects. The motion profile for the training of this network is a movement with a constant velocity. This velocity is $v=0.025$

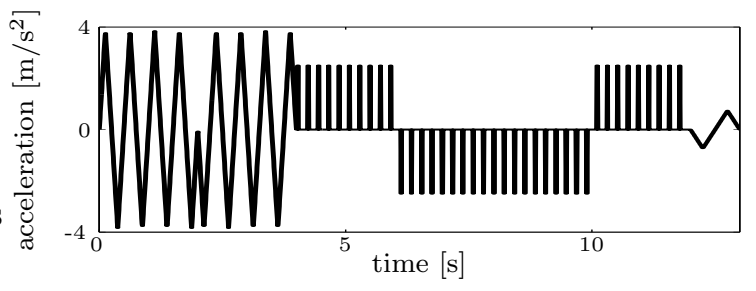

Fig. 3. Motion profile for the learning of the mass

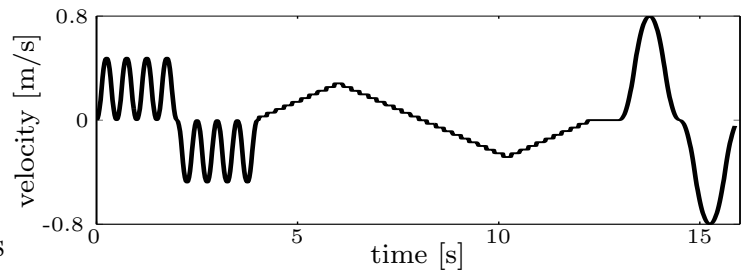

Fig. 4. Motion profile for the learning of friction

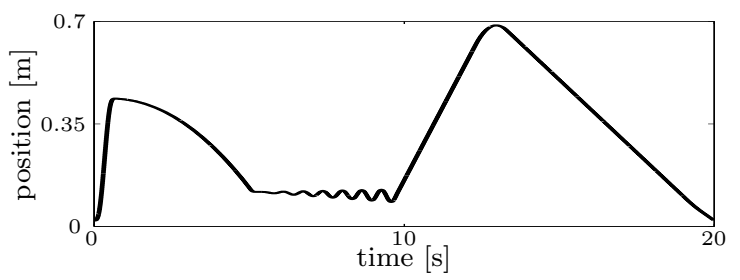

Fig. 5. Evaluation path

$[\mathrm{m} / \mathrm{s}]$ to make sure that the position dependent effect fell well within the bandwidth. The positiondependent network is trained before the velocitydependent network because the friction is approximately point symmetric in zero. This means that a constant term is added if the movement is made in one direction and this same constant term is subtracted if the movement is made in opposite direction. The last network that is trained is the network that should compensate for the friction. The motion profile is given in figure 4. This motion features a broad set of velocities at different positions.

To test if the networks contain the correct functions, a motion is performed that has not been used during the training. This motion is given in figure 5. This motion contains fast accelerations at the beginning to test if the mass is correctly learnt. After this, an oscillatory movement is done to test if the friction is correctly approximation around small values for the velocity. The evaluation motion ends with a slow movement to test if the cogging is learnt correctly.

\section{RESULTS}

The experiments have been carried out for the four different configurations and the results for the evaluation motion are given in the figures 6 to 9 . In these figures the gray line is the tracking error before the learning, i.e. PD control only, and the black line is the tracking error after learning. In all the cases the tracking error reduces significantly. 


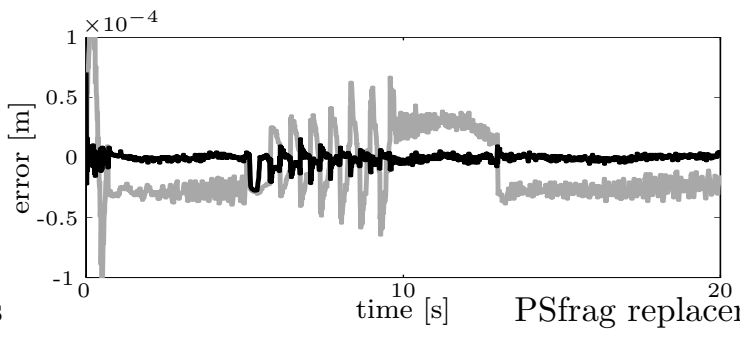

Fig. 6. Tracking error for good magnets and complete comb

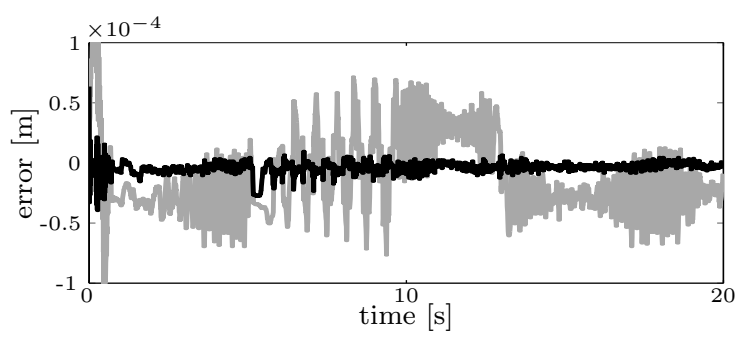

Fig. 7. Tracking error for bad magnets and complete comb

Please note the difference in scale between the first two and the last two figures.

From these figures several observations can be made. First, it can be seen that the tracking error is relatively large if the velocity changes sign. This happens several times between $t=5$ and $t=10[\mathrm{~s}]$. The friction changes approximately discontinuously if the velocity changes sign due to the Coulomb effect. The function approximator is not capable of approximating this fast change due to the width of the splines. It is of no use to decrease the width of the splines because the hysteresis would make it impossible to learn a correct value.

Second, it can be seen that the error of fast movements is larger than for slow movements if the outer-teeth are missing. In figure 6 and 7 there is no difference between the fast movement from $t=10-13[\mathrm{~s}]$ and the slow movement at $t=13-$ $20[\mathrm{~s}]$. While in figure 8 and 9 a difference in error can be seen. This shows that forces introduced by the absence of the outer-teeth of the comb cannot be compensated for by a separate network for position-dependent effects and a network for the velocity-dependent effects.

Other observations are that an error due to acceleration is not present after learning, so the mass is learnt well. Furthermore, the constant error due to the use of a PD controller for constant movements is removed. The last seven seconds of figures 6 and 7 beautifully illustrates the influence of the magnet placement and properties on the cogging characteristics.

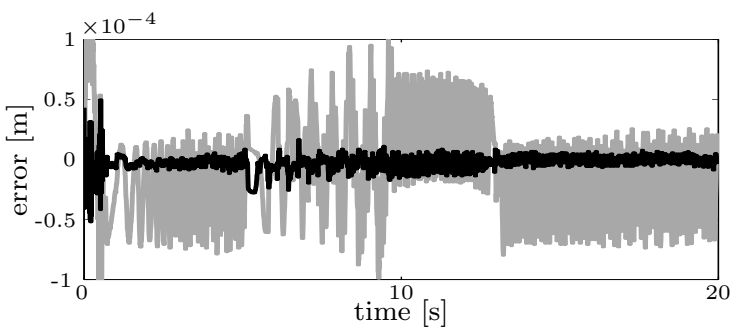

Fig. 8. Tracking error for good magnets and incomplete comb

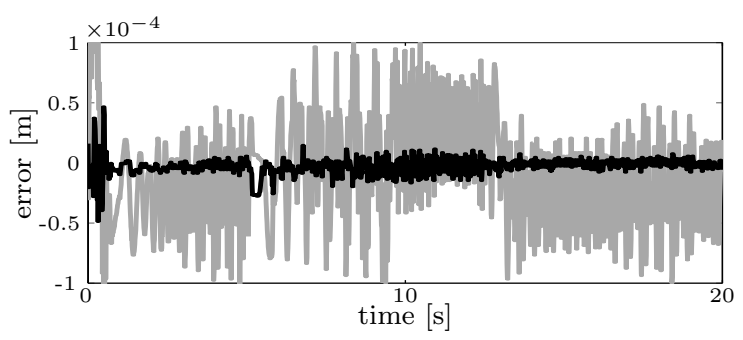

Fig. 9. Tracking error for bad magnets and incomplete comb

\subsection{Network contents}

4.1.1. Acceleration The acceleration network had to estimate the mass of the translator. The mass of the translator with the outer-teeth was estimated on 10.5 and $10.7[\mathrm{~kg}]$ for respectively the good and the bad magnet plate. The small difference can be contributed to crosstalk from the position network. In the experiment with the high cogging force, the error that is contributed to the unknown mass could be originated from a position dependent effect, thus introducing a value for the mass slightly different.

The mass of the translator without the outer-teeth was twice estimated near 11 [kg]. This translator was a different one, which could explain for the difference in mass. It might also be contributed to crosstalk from other effects.

4.1.2. Velocity The velocity network has to compensate for the friction. The contents of this network is depicted in figure 10. The contents for the different configurations look similarly and are not given here. The contents of the network is extrapolated linearly for the outer regions. The figure shows what is expected for the friction characteristic. The stiction can be seen at the zero velocity crossing. The viscous friction, the Coulomb friction and the Stribeck effect can be observed in this figure.

4.1.3. Position The position network had to compensate for all the effects that are positiondependent. The contents of this network is heavily influenced by the quality of the magnets as well as by the absence of the outer-teeth. The contents of this network is given in the figures 11 to 14 . 


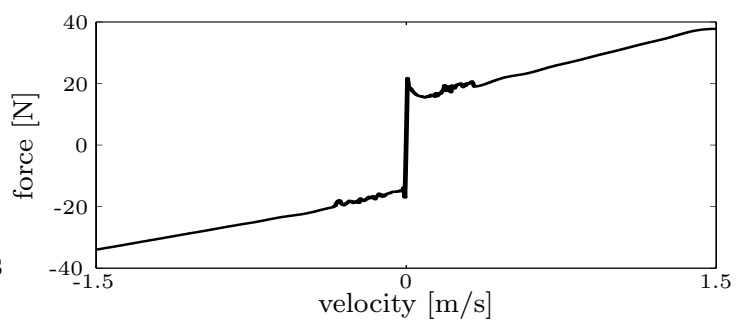

Fig. 10. Contents of velocity network

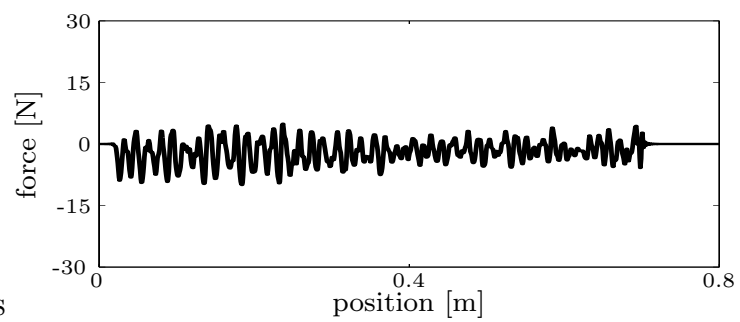

Fig. 11. Position network for good magnets and complete comb

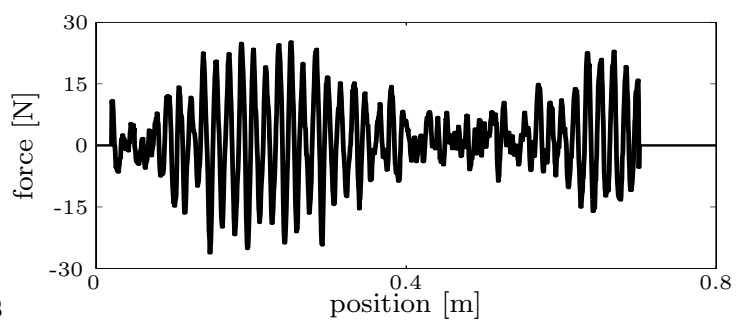

Fig. 12. Position network for bad magnets and complete comb

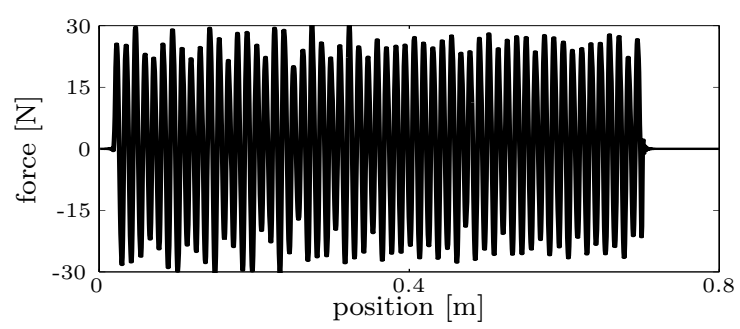

Fig. 13. Position network for good magnets and incomplete comb

In figure 11 it can be seen that for the good configuration the cogging force is not too large. It stays within -10 and $+5[\mathrm{~N}]$. When the good magnet plate is replaced by the bad magnet plate, it can be seen that the force that is required to compensate for the cogging force is much higher. This is illustrated in figure 12 .

The contents of the position network for good magnets and without the outer-teeth is given in figure 13. The force that is required to compensate for the position dependent effects is increased significantly by the absence of the outer-teeth. In figure 14 it can be seen that the force to compensate for the position dependent effect is largest if next to the comb without the outer-teeth the bad magnet plate is used.

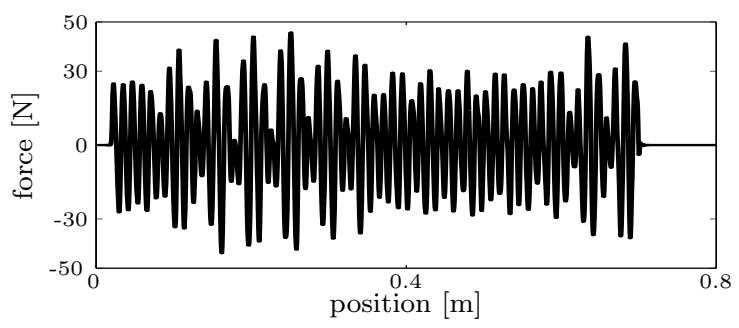

Fig. 14. Position network for bad magnets and incomplete comb (note the scale!)

\section{CONCLUSION}

In this paper it is shown by experiments that the Learning Feed-Forward setting is capable of significantly decreasing the tracking errors of a motion system. It does so by approximating the inverse of the plant including the non-linear statedependent effects like friction and cogging. It is possible to use feed-forward control to compensate for tracking errors that are introduced by lowcost construction. This makes it attractive to use low-cost control techniques instead of expensive construction, cutting down the development and production cost. For all configurations that were considered controlled by LFF, the performance was better than the configuration with the good magnet plate and the inclusion of the outer-teeth controlled only by PD control.

\section{REFERENCES}

Brown, M. and C. Harris (1994). Neurofuzzy adaptive modeling and control. Prentice Hall International. UK.

de Kruif, B.J. and T.J.A. de Vries (2001). On using a support vector machine in learning feed-forward control. In: Proc. Int. Conf. On Advanced Intelligent Mechatronics (AIM'01). IEEE/ASME. Como,Italy. pp. 272-277.

de Vries, T.J.A., W.J.R. Velthuis and L.J. Idema (2001). Application of parsimonious learning feedforward control to mechatronic systems. IEE Proc. D: Control Theory $\&$ Applications 148(4), 318-322.

Gieras, J.F. and Z.J. Piech (2000). Linear Synchronous Motors, Transportation and Automation Systems. CRC Press. Boca Raton, Florida.

Otten, G., T.J.A. de Vries, J. van Amerongen, A.M. Rankers and E.W. Gaal (1997). Linear motor motion control using a learning feedforward controller. IEEE/ASME Trans. Mechatronics 2(3), 179-187.

Velthuis, W.J.R. (2000). Learning Feed-Forward Control, Theory, Design and Applications. PhD thesis. University of Twente. Enschede.

Yao, B. and L. Xu (2002). Adaptive robust motion control of linear motors for precision manufacturing. Mechatronics 12, 595-616. 